\title{
The Multipoles Factory: An Element of the LHC Control
}

\author{
S. Amet, L. Bottura, L. Deniau, and L. Walckiers
}

\begin{abstract}
The measurements performed at CERN on prototypes and first pre-series main dipole magnets confirm the need of an active control of the Large Hadron Collider to compensate the dynamic field changes during the proton beam injection and acceleration. This control requires in turn an accurate forecast of the magnetic field in the accelerator. We plan to predict the field on the basis of two elements: theoretical field models tailored through the accumulated knowledge of the main magnets during series tests, and an on-line measurement system running on few reference magnets tracking the $\mathrm{LHC}$ current cycle. Data coming from this "Multipoles Factory" will result from the fusion of the two sources. Based on this system we foresee to deliver calibration information for pre-defined accelerator cycles as well as real time information for the active control. In this paper we report the conceptual design of the system, and we discuss the features and performance of the models that we have developed for the field forecast.
\end{abstract}

Index Terms-Accelerator control, accelerator magnets, magnet simulation, magnetic measurements.

\section{INTRODUCTION AND NOTATIONS}

$\mathbf{T}$ HE STABILITY and the reproducibility of the field strength and quality of the LHC superconducting magnets have been extensively measured on prototypes and first pre-series magnets. The constrains on the field quality [1], [2] has lead to design a Multipoles Factory (MF) as an important element of the LHC control.

The inputs (left side of Fig. 1) to be supplied by the LHC control operation will be the time $t$ from the Global Positioning System clocks, the temperature $T$ and the operating current $I$ ( $d I / d t$ will be computed from the current history). For our purposes, we will only consider the currents such that $I_{i n j} \leq I \leq$ $I_{n o m}$. The specific currents of the LHC are the injection current $I_{i n j}=760 \mathrm{~A}$, the nominal current $I_{\text {nom }}=11850 \mathrm{~A}$, the ultimate current $I_{u l t} \approx 13 \mathrm{kA}$ and the critical current $I_{c} \approx 14 \mathrm{kA}$ (maximum current sustainable by cables at nominal field and $1.9 \mathrm{~K})$.

The outputs (right side of Fig. 1) are expressed in term of the complex field harmonics $\mathbf{C}_{n}=B_{n}+\mathbf{i} A_{n}$ in Tesla where $B_{n}$ is the $n$th normal harmonic and $A_{n}$ is the $n$th skew harmonic in the main field harmonic frame $B_{N}$, (e.g., $A_{N}=0$ ). The magnet field can be expressed by [3]:

$$
B_{y}+\mathbf{i} B_{x}=\sum_{n=1}^{\infty} \mathbf{C}_{n}\left(\frac{\mathbf{z}}{R_{r e f}}\right)^{n-1}
$$

Manuscript received September 24, 2001.

The authors are with the CERN European Organization for Nuclear Research, CH-1211 Geneva 23, Switzerland (e-mail: Laurent.Deniau@cern.ch).

Publisher Item Identifier S 1051-8223(02)04210-0.

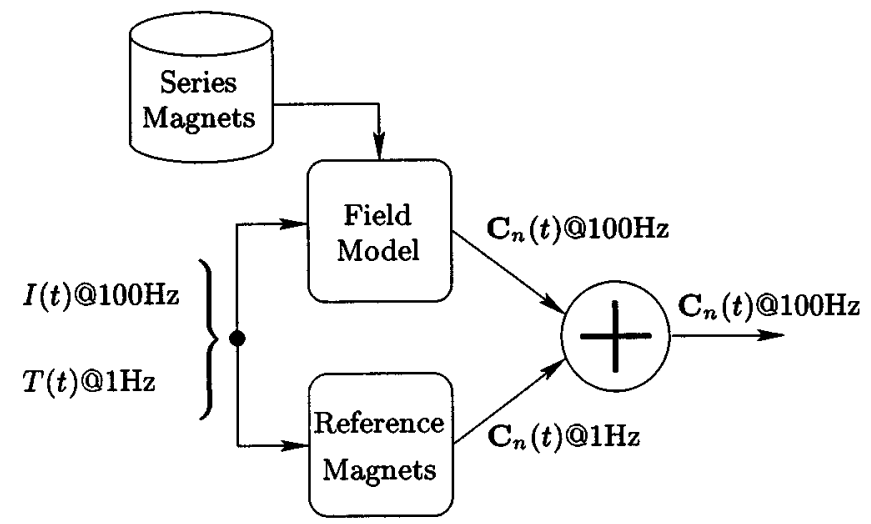

Fig. 1. Schematic representation of the Multipoles Factory. Operation current and temperature are inputs (left side) of the system while the outputs are the complex field harmonics estimation (right side). The sampling frequencies are given in Hertz for each data flows.

with $R_{\text {ref }}=0.017 \mathrm{~m}$ and where $n=1$ for dipole field, $n=2$ for quadrupole field, $n=3$ for sextupole field, etc.

\section{THE Multipoles FACTORY}

The main application of the MF is to provide an estimation of the machine magnetic state, good enough to inject and keep the beam until the beam instrumentation (online measurement) takes over. Another purpose of the MF is to provide an estimation of the harmonics which will not be measured but for which correctors exist (e.g., $A_{3}, B_{4}, B_{5}$ ).

As shown in Fig. 1, the MF is composed of four different elements:

- The series magnets database stores the digested experimental data on the LHC magnets archived during the series measurements.

- The field model represents a condensed summary of the mathematical and physical knowledge of the series measurements based on the series magnets database. It provides a theoretical estimation of the machine magnetic state at medium frequency. It will be extensively described in a later section.

- The reference magnets are magnets tracking the LHC operation cycle while being measured online. They provide an experimental estimation of the machine magnetic state at low frequency.

- The data fusion operator $\bigoplus$ is a sophisticated adaptive algorithm able to merge together the experimental and model estimation to get the best machine magnetic state 
estimation. This operator will also be able to predict approximately the machine magnetic state for a given time in advance.

The MF can be used in two different modes:

- Dynamic Engine: With the reference magnets connected and the LHC current and temperature as real time inputs, the MF is able to provide an estimation of the magnetic state of the machine for each magnet (local), magnet family (e.g., dipoles, quadrupoles and correctors) and machine sector (integrated). From the harmonics forecast, the field errors can then be converted online into trims of currents for the power supplies of correctors magnets.

- Static Engine: Without the reference magnets connected but using any desired LHC operation cycle (like Fig. 2) as input, the MF is able to forecast the harmonics for each magnet, magnet family and machine sector. The harmonics forecast can then be converted into tables of currents to be downloaded off-line to the power supplies of each magnet family.

\section{THE LINEAR FIELD MODEL}

One of the key elements of the MF is the field model that we will use to forecast field values as a function of operation conditions. We have started development using data from pre-series magnets. The field model presented in this paper balances the necessity to fit inside the requirements the experimental data from the series measurements performed on the LHC series magnets against the wish to stay close enough to physical models. The model does not consider explicitly the nonlinearity of some parameters (i.e., history, quenches, temperature).

For a given normal harmonic $B_{n}$ and considering only linear contributions, the model has the form:

$$
B_{n}(t, I, d I / d t)=B_{n}^{D C}+B_{n}^{A C S}+B_{n}^{A C L}
$$

where $B_{n}^{D C}$ is the direct current components, $B_{n}^{A C S}$ is the alternate current components for short term effects $(<1 \mathrm{~s})$ and $B_{n}^{A C L}$ is the alternate current components for long term effects $(>100$ s). The model is also valid for any skew harmonic $A_{n}$.

\section{A. Quasi Steady State}

The quasi steady state contribution ( $D C$ component) is present in all phases of the LHC operation cycle [phases i)-v) of Fig. 2]. The quasi steady state model is further decomposed as follow:

$$
B_{n}^{D C}=B_{n}^{\text {res }}+B_{n}^{g e o}+B_{n}^{d i s}+B_{n}^{\text {mag }}+B_{n}^{\text {sat }}
$$

where the constituents are explained thereafter.

1) Residual Magnetization Contribution: The residual magnetization is coming from the permanent magnetization of some magnet components (e.g., the iron yoke) and can be important for small excitations. It is sensitive to the magnet history.

Model:

$$
B_{n}^{r e s}=\alpha_{n}^{r e s} .
$$

2) Geometric Contribution: The largest part of the field in the LHC magnets is generated by the current in the supercon-

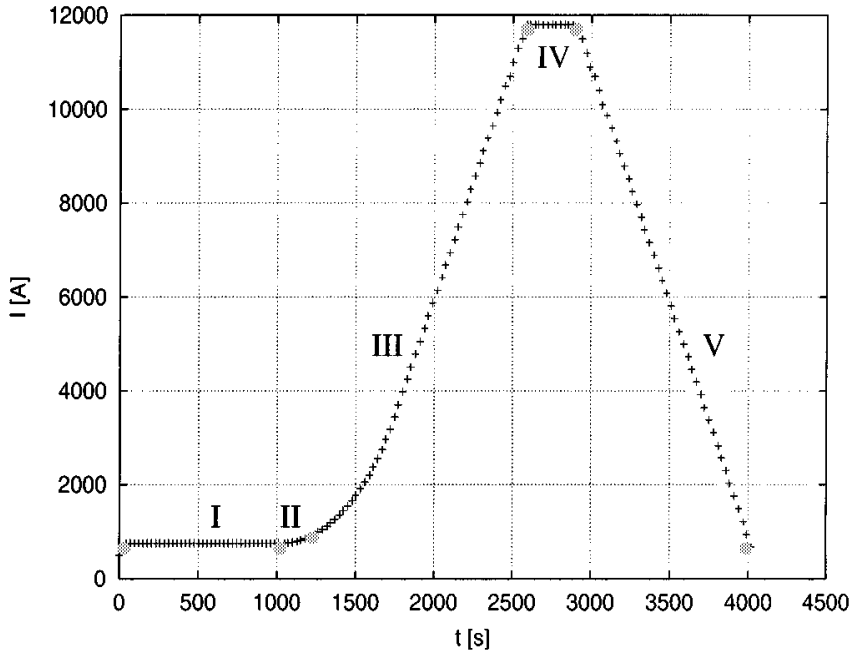

Fig. 2. Typical LHC operation cycle. The dots separate the cycle phases used to estimate the parameters of the linear model: i) injection, ii) ramp start (snapback), iii) ramp up (acceleration), iv) flat-top (physics), v) ramp down.

ducting cables. A minor portion is due to the magnetization of the iron yoke or the other magnetic components (e.g., collars). We define the geometric contribution as the linear part of the field for the main harmonic. For higher harmonics, it is associated with the deviations from the ideal current distribution producing only the main harmonic. It is sensitive to the powering sequence of the magnet (i.e., maximum Lorentz force sustained).

Model:

$$
B_{n}^{g e o}=\alpha_{n}^{g e o} I .
$$

3) Displacement Contribution: The displacement contribution is coming from the elastic movements of the cables within the coil cross section due to the applied Lorentz forces.

Model:

$$
B_{n}^{d i s}=\alpha_{n}^{d i s} I^{3} .
$$

4) DC Magnetization Contribution: This contribution originates from the DC magnetization of the filaments in the cables strands (i.e., persistent currents) and is significant at low field, where the superconductor magnetization is higher. It depends on the current cycle (i.e., hysteresis) and temperature.

We take for this contribution a model that has been found to fit well the critical current behavior as a function of the background field [4]

Model:

$$
B_{n}^{m a g}= \pm \alpha_{n}^{m a g}\left(\frac{I}{I_{c}}\right)^{n_{0}}\left(1-\frac{I}{I_{c}}\right)^{n_{1}}
$$

where the + is for the ramp up and - for the ramp down.

Parameters:

$$
\alpha_{n}^{m a g}, \quad n_{0} \approx-2.5, \quad n_{1} \approx 1 .
$$

5) Saturation Contribution: The saturation contribution is coming from the changes of the magnetic permeability in the iron yoke surrounding the coils at high field. 
Although saturation effects can be modeled using magnetostatic codes, the associated effort is large. We have chosen to describe this effect with a simplified model composed of two approximations of the step function which together fit the data while remaining simple enough for the optimization algorithm.

Model:

$$
B_{n}^{s a t}=\left[\alpha_{n}^{s a t 1} f_{1}(I)+\alpha_{n}^{s a t 2} f_{2}(I)\right] I
$$

where

$$
f_{k}(I)=\frac{1}{\pi}\left[\arctan \left(\frac{I-I_{n}^{k}}{\Delta I_{n}^{k}}\right)+\frac{\pi}{2}\right], \quad k=1,2 .
$$

Parameters:

$$
\alpha_{n}^{\text {sat } 1}, \quad I_{n}^{1}, \quad \Delta I_{n}^{1}, \quad \alpha_{n}^{\text {sat } 2}, \quad I_{n}^{2}, \quad \Delta I_{n}^{2} .
$$

\section{B. AC Short Term Effects (Coupling Currents)}

The coupling currents contribution is due to interfilamentary currents within the strands and interstrands currents within the cables during the ramp up and down cycles. For the typical ramp times to be used in the LHC operation, they can be assumed to be fully developed in resistive regime, that is all inductive and shielding effects have already decayed. The model includes the eddy currents in other conducting parts (but does not consider them separately) and it assumes the interstrands resistance to be constant. The magneto-resistance (i.e., $B$-dependence) is not taken into account.

Model:

$$
B_{n}^{A C S}=\alpha_{n}^{A C S} \frac{d I}{d t}
$$

\section{AC Long Term Effects (Decay and Snapback)}

These effects are measured during a long plateau followed by a ramping start [phases i) and ii) of Fig. 2]

$$
B_{n}^{A C L}=B_{n}^{\text {dec }}+B_{n}^{s n a} .
$$

1) Decay: The field decay is attributed to magnetization changes. It is sensitive to the history and quenches of the magnet.

The basis of the model is our present understanding of the physics underlying this decay. We believe that decay is associated with the changes in the internal field due to current diffusion. Under drastic simplifications, the latter has an analytic solution obtained as a series of exponentials considered here up to the third term [5].

$$
\begin{aligned}
& \text { Model: } \\
& \qquad B_{n}^{\text {dec }}=\sum_{k=1}^{3} \alpha_{n}^{\text {deck }}\left(1-\exp \left(-\frac{(2 k-1)^{2}\left(t-t_{0}\right)}{\tau_{0}}\right)\right) .
\end{aligned}
$$

\section{Parameters:}

$$
\alpha_{n}^{d e c 1}, \alpha_{n}^{d e c 2}, \alpha_{n}^{d e c 3}, \tau_{0}>0 .
$$

2) Snapback: The snapback occurs during the rapid re-establishment of the magnetization (ramp start) after the decay of a constant current plateau. It is important mainly at the beginning of the ramp where the beam stability is most critical.

This model does not come from any physical model, but it fits the data with a minimum of parameters.

Model:

$$
B_{n}^{s n a}=\sum_{k=1}^{2} \alpha_{n}^{\text {snak }} \exp \left(\beta_{n}^{k}\left(I-I_{i n j}\right)\right), \quad I>I_{i n j} .
$$

Parameters:

$$
\alpha_{n}^{s n a 1}+\alpha_{n}^{s n a 2} \approx B_{n}^{\text {dec }}, \beta_{n}^{1}, \beta_{n}^{2} .
$$

\section{The Model Evaluation}

The model estimation is performed step by step on measurements taken during an LHC operation cycle (except for the coupling current estimation) where for each step, some of the 21 parameters are estimated from selected measurements and fixed for the following steps [6]:

1) The coupling currents estimation is performed on a special set of four ramps up and down between $I_{i n j}$ and $I_{\text {nom }}$ at the ramp rates 10,20,40 and $50 \mathrm{~A} / \mathrm{s}$. After having removed the geometric components we get $\alpha_{n}^{A C S}$ from linear fits.

2) The saturation estimation is achieved from phases iii) and v) of the LHC cycle (cf. Fig. 2) without the ramp effects $B_{n}^{A C S}$. We take $I_{n}^{1}, \Delta I_{n}^{1}, I_{n}^{2}$ and $\Delta I_{n}^{2}$ from nonlinear fits.

3) The DC magnetization is estimated from phases iii) and v) of the LHC cycle without the geometric components, the ramp effects and the saturation contribution. We take only $n_{0}$ and $n_{1}$ from nonlinear fits.

4) The full estimation of $B_{n}^{D C}$ is then performed on the phases iii) and v) of the LHC cycle using linear fits with the previously estimated parameters fixed.

5) The snapback is estimated from phases ii) (i.e., ramp start) without the DC components. We take $\alpha_{n}^{\text {sna1 }}, \alpha_{n}^{\text {sna } 2}$, $\beta_{n}^{1}$ and $\beta_{n}^{2}$ from nonlinear fits.

6) The decay estimation is performed from phases i) (i.e., constant current) with the offset $B_{n}^{D C}\left(I_{i n j}\right)$ subtracted. We take $\alpha_{n}^{d e c 1}, \alpha_{n}^{d e c} 2, \alpha_{n}^{d e c 3}$ and $\tau_{0}$ from nonlinear fits.

\section{The Mode Performance}

To demonstrate the performance of the model presented here, we have plotted in Figs. 3-5 a comparison of measured and modeled harmonics during an operation cycle of the type shown in Fig. 2. The measurements were performed in the first pre-series dipole. The plots show the dipole transfer function and the first two allowed harmonics, sextupole and decapole as a function of time.

The data are described accurately, and the model has the advantage to provide naturally a smoothing of measurement errors as seen in the case of the transfer function at low current where 


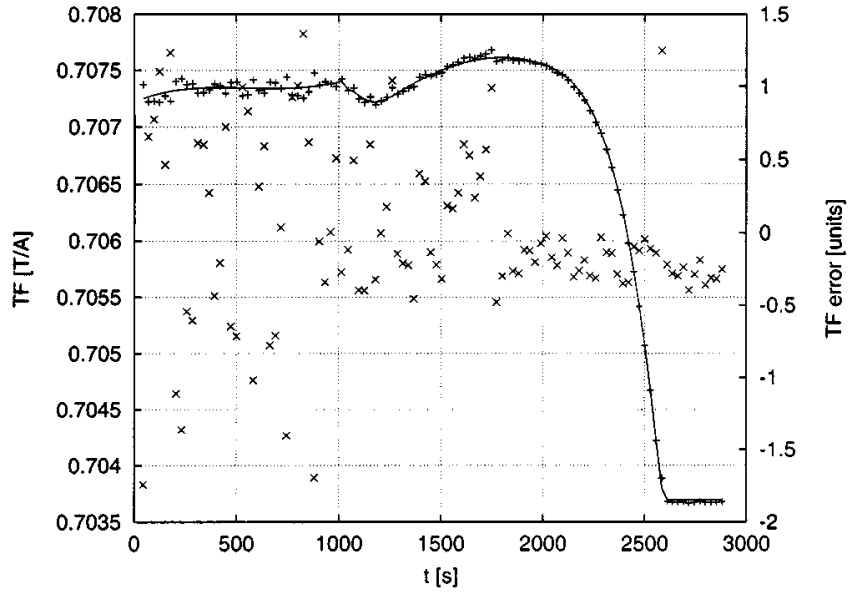

Fig. 3. Transfer function data $(+)$, model (line) and errors $(x)$.

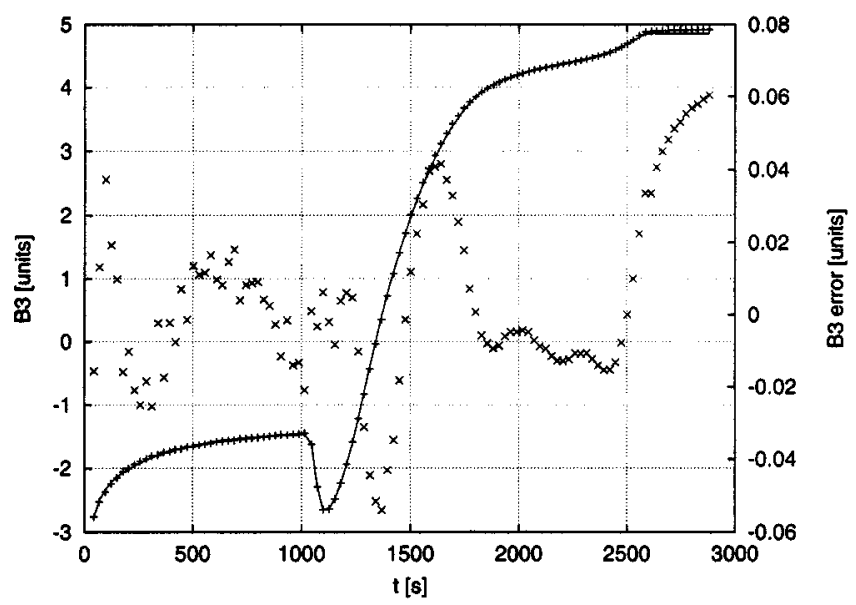

Fig. 4. $B_{3}$ data $(+)$, model (line) and errors $(\times)$.

the measurement resolution becomes visible. In Figs. 3-5 we have plotted also the local error defined as the difference between measurement and model. The overall errors in the modeled transfer function are within 1.5 unit on the transfer function, better than 0.06 unit for the sextupole and 0.015 unit for the decapole harmonics. These behaviors are also valid for the other harmonics. The small but nonnegligible decay effect during operation plateau for harmonics $B_{3}(\approx 0.06$ unit $)$ and $B_{5}(\approx 0.008$ unit) can also be modeled on request by equation (12) using the starting time of phase iv) as $t_{0}$ and $B_{n}^{D C}\left(I_{n o m}\right)$ as offset. The summary of the model performance is shown in Fig. 6 for low-order harmonics important for accelerator operation and where the expected requirements are satisfied if the maximum local error stays below the LHC operation tolerances.

In general the model allows data interpolation with a maximum local error within the range of 0.01 to 0.1 unit. The RMS error is better by roughly one order of magnitude. For the main harmonic $B_{1}$ the required integrated error is two orders of magnitude smaller than the achievable field error in the dipoles and one order of magnitude smaller than the model error. The MF does not therefore fill the accuracy gap to provide the requested corrections, and direct beam measurements will remain the only mean to fully correct for $B_{1}$ and $B_{3}$ errors. On other harmonics

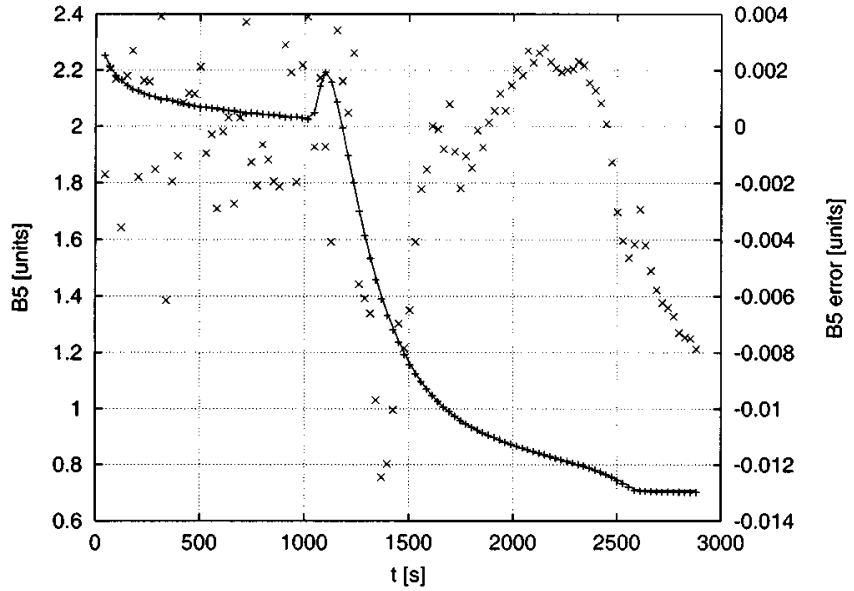

Fig. 5. $B_{5}$ data $(+)$, model (line) and errors $(\times)$.

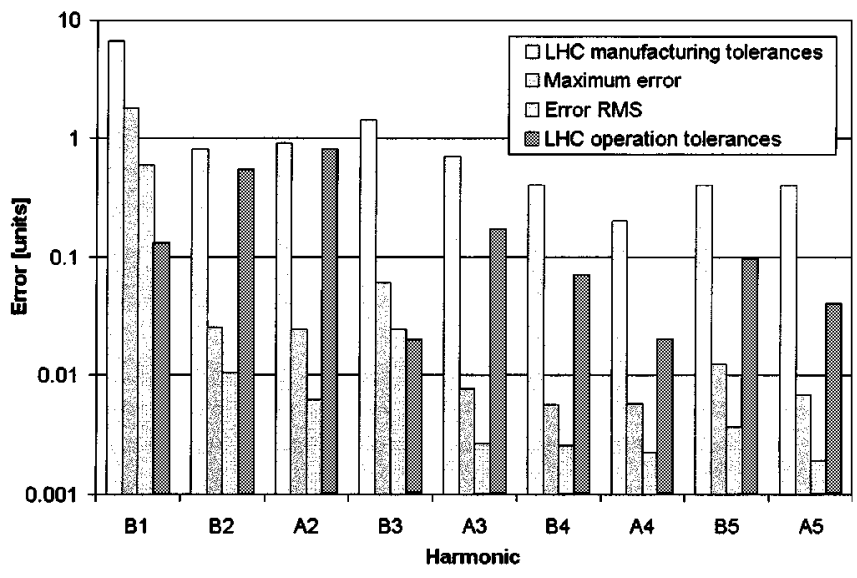

Fig. 6. Harmonics errors between model and experimental data versus manufacturing tolerances and operation tolerances.

the model error is generally better than the accelerator tolerances, and the MF can therefore be a viable source for the corrections. This applies in particular to harmonics of higher order such as $B_{4}$ and $B_{5}$, for which direct beam measurements could be difficult.

\section{CONCLUSION}

We have described an interpolation model for the field in a LHC dipole. The model can reproduce with high accuracy the behavior of the main field and its harmonics, thus providing a good basis for the LHC Multipoles Factory. The accuracy of the model is such that it could be used for direct correction of harmonics like the normal octupole and decapole within specified values for LHC operation. In addition the model can provide correction values and reduce field errors by one order of magnitude, thus also decreasing the required dynamics of the feed-back system for LHC control. The next step will be to address important issues such as nonlinearity and nonreproducibility in the model parameters. This type of effect is known to be associated with operating temperature variations, magnet cycling and powering history which will be studied during the tests of the LHC series magnets. 


\section{REFERENCES}

[1] L. Walckiers, "Steering field and current in the LHC magnets," in Chamonix XI Proc., Jan. 2001.

[2] R. Bailey et al., "Dynamic effects and their control at the LHC," in PAC'97, May 1997.

[3] R. Wolf, "Field error naming conventions for LHC magnets," LHC Project Engineering Specification, August 1998.
[4] L. Bottura, "A practical fit for the critical surface of NbTi," IEEE Trans. Appl. Superconduct., vol. 10, no. 1, pp. 1054-1057, 2000.

[5] L. Krempasky and C. Schmidt, "Experimental verification of supercurrents in superconducting cables exposed to AC-fields," Cryogenics, vol. 39, pp. 23-33, 1999.

[6] S. Amet, L. Bottura, and L. Deniau, "Standard analysis procedures for field quality measurement of the LHC magnets," LHC Project Notes, 1998-2001. 